\title{
Extraction of zinc metal ions from aqueous solution using ionic liquids
}

\author{
Tariq S.M. ${ }^{1, *}$, HanHui Z. ${ }^{1, *}$, Yang J. ${ }^{2}$, Ibrahim M.. ${ }^{3}$, Wang L. ${ }^{1}$ and Tasleem A. ${ }^{4}$ \\ ${ }^{1}$ School of Environment Science and Spatial Informatics, China University of Mining and Technology, Xuzhou, P.R China 221116 \\ ${ }^{2}$ Nanjing Research Institute of Environmental Science, Nanjing, 210042, P.R. China \\ ${ }^{3}$ Department of Environmental Sciences and Engineering, Government College University, Allama Iqbal Road, Faisalabad Pakistan \\ ${ }^{4}$ Institute of Pharmaceutical Sciences, University of Veterinary and Animal Sciences, Lahore, 54000, Pakistan \\ Received: 13/08/2020, Accepted: 14/12/2020, Available online: 22/12/2020 \\ *to whom all correspondence should be addressed: e-mail: tariqsarwar98@yahoo.com, zhanhhh@263.net \\ https://doi.org/10.30955/gnj.003434
}

\section{Graphical abstract}

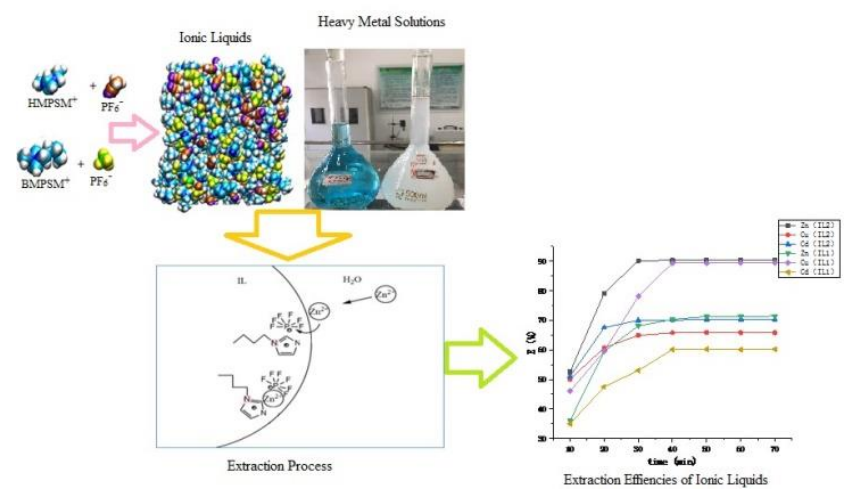

\section{Abstract}

This work analyzes the extraction behavior of transition heavy metal Zinc and other metal ions such as Copper, and Cadmium from hydrochloride aqueous solution in the absence of chelating agents by using a novel class of hydrophobic ionic liquids. Ionic liquid for this study was synthesized based on 1-(n-alkyl)-3-methylimidazolium cations and hexafluorophosphate anions followed by the metathesis route at room temperature were evaluated. The advantages of using these ionic liquids include their simple synthesis and sustainability. Which makes them "Greener and Simpler" compared to other solvents used for metal extraction. The experimental results show that ionic liquid 1-Butyl-3-methylimidazolium hexafluorophosphate, [BMPSM][PF 6 ] entirely removed of Zinc (extraction percentage 94.4\%) and other Copper and Cadmium heavy metals from the aqueous solutions. Based on the results, the use of ionic liquids for selected heavy metal ions as a substitute to the traditional extraction agents in liquid/liquid extraction of heavy metal ions shows considerable potential and is quite promising and promoting for further extraction.

Keywords: Heavy metals, wastewater, ionic liquids, metal ions extraction.

\section{Introduction}

Over the past few decades, disproportionate population growth and rapid development of industries culminated severe wastewater pollution containing heavy metal ions affecting natural water bodies (Kenntner et al., 2003). The presence of traces of heavy metals in wastewater and industrial effluents is one of the major concerns of environmental pollution (Babel and Kurniawan, 2004). Heavy metal pollution is considered to be more damaging than other sorts of water pollution as it causes serious ill effects because due to high degree of toxicity (Li and Trost, 2008; Tian et al., 2019). Therefore, the decontamination of wastewater is an area of enormous focus in the field of environmental waste and pollution control (Nordberg and Nordberg, 2009).

Heavy metals elimination by traditional processes for industrial aqueous effluents includes electrolysis, ion exchange, chemical precipitation, solvent extraction, membrane separation, coagulation and adsorption (Blais et al., 1999; Fu and Wang, 2011). Among these technologies, liquid-liquid extraction is one of the efficient and simple techniques to separate and concentrate metal ions from the industrial wastewaters (Singh and Savoy, 2020).

As a matter of fact, technologies are important for heavy metals removal from wastewater and impediment to their secretion into surface water (Gunatilake, 2015). The use of ionic liquids as extracting agents for the removal of heavy metals from the wastewater could be an excellent alternative to conventional procedures (Faur-Brasquet et al., 2002; Pacheco-Fernández and Pino, 2019).

Ionic liquids are environment-friendly compared with volatile organic solvents because of undetectable vapor pressure and good chemical and thermal stability at high temperature (Sheldon, 2001). Recently ionic liquids are considered in a broad variety of chemical and biochemical processes as a clean alternate to typical organic solvents (He et al., 2020; Toral et al., 2007). Additionally, the properties of ionic liquids (solubility, hydrophobicity, viscosity, etc.) can be diversified by flashing the substituent 
group on the cation or combined anion, making them more suited for the use as "green designer" solvents (Caparica et al., 2018; Gardas and Coutinho, 2008). This prominent characteristic plays a key role in realizing the successful extraction processes since proper combinations of cation and anion of the solvents can be made (Ghandi, 2018) (Ríos et al., 2009). Ionic liquids have recently been introduced as attractive clean alternatives to traditional organic solvents in an extensive range of chemical and biochemical processes (Toral et al., 2007; Vekariya, 2017). They also function effectively in extraction processes, such as separation of metal species (Germani et al., 2007; Marsousi et al., 2019), organic compounds (Marsousi et al., 2019), and even macromolecules (Marsousi et al., 2019). Over the past few years, extraction of several metal ions using ionic liquids containing suitable complexing agents such as crown ether (Marsousi et al., 2019), dithizone (Phuong et al., 2010), and other organic ligands has been carried out (Isosaari et al., 2019; Zhang et al., 2018).

Examples of 1-(3-pyridyl) undecan-1-one oxime extractor and its quaternary salts have been successfully tested as possible Zn extractors (Wieszczycka, 2013). Efficient removal of copper from chloride solutions by bis(isodecyl)pyridine-3,5-dicarboxylate which has been successfully used as an attractive extractant. The most interesting extractant is 1-(2-pirydyl) tridecan-1-one oxime, which, as an extremely complexing agent, has the ability to extract copper effectively. Laboratory-scale studies demonstrate that 3-[1-(hydroxyimine) undecyl]-1propylpyridinium bromide is effective and versatile in copper extraction (Wieszczycka et al., 2012; Wojciechowska et al., 2017b). Another example of successfully $\mathrm{Pb}$ (II) extraction via chloride formulations using Ionic Liquid 3-[1-(hydroxyimine) undecyl]-1propylpyridinium chloride (3PC10-PrCl) dissolved in methyl isobutyl ketone (MIBK) (Wojciechowska et al., 2017a).

Phosphonium ionic liquids such as trihexyl (tetradecyl) phosphonium chloride (Cyphos IL 101), trihexyl (tetradecyl)phosphonium bis(2,4,4-trimethylpentyl) phosphinate (Cyphos IL 104) and tributyl(tetradecyl) phosphonium chloride (Cyphos IL 167) as extractants selective extraction of ruthenium(III) in the existence of $\mathrm{Rh}$ (III) acidic aqueous solutions was successfully evaluated (Rzelewska et al., 2017).

Efficiently removal of cobalt, manganese, iron and zinc eliminated from rare earths and nickel in metal-filled liquor with a high concentration of chloride ( $8 \mathrm{M}$ chloride) by ionic liquids trihexyl(tetradecyl)phosphonium chloride (Cyphos IL 101) or tricaprylmethylammonium chloride (Aliquat 336) (Larsson and Binnemans, 2014).

Four separate TSILs used as an extractor: Trioctylmethylammonium bis(2-ethylhexyl) phosphate [TOMA][D2EHP]; trioctylmethylammoniumbenzoate [TOMA][BA]; trihexyltetradecylphosphonium bis(2ethylhexyl) phosphate $\left[\mathrm{P}_{6,6,6,14}\right][\mathrm{D} 2 \mathrm{EHP}]$; and trihexyltetradecylphosphonium benzoate $\left[\mathrm{P}_{6,6,6,14}\right][\mathrm{BA}]$ were found to have high molybdenum (VI) extraction rates. In comparison, the best cation was [TOMA]+ for both diluents and $[\mathrm{BA}]-$ was the best anion while kerosene and 1-octyl-3-methylimidazolium bis(trifluoromethylsulfonyl)imide, [omim][Tf2N] were used as diluents (Quijada-Maldonado et al., 2018). Additionally, using two ionic liquids, 1-octyl-3-methylimidazolium bis(trifluoromethylsulfonyl)imide ([OMIM][NTf2]) and trihexyltetradecylphosphonium bromide (Cyphos 102) successfully investigated for separating Pt(IV) and Pd(II) in acidic aqueous solution containing $\mathrm{HCl}$ (Papaiconomou et al., 2015). This was a reason to continue the equilibrium extraction studies of the most widely related metals using an oxygen-containing solvent reagent as an organic diluent.

In this work, using two different imidazolium-based ionic liquids, 1-Hexyl-3-methylimi-dazolium hexafluorophosphate (IL1) and 1-Butyl-3-methylimidazolium hexafluorophosphate (IL2) were synthesized and tested for the extraction of transition heavy metal especially zinc and other metals such as copper and cadmium from aqueous hydrochloride solutions in the absence of chelating agent at room temperature. Also, the extraction percentage (\%E) of metal ion concentrations by the influence of ionic liquids was evaluated.

\section{Experimental section}

\subsection{Reagents}

The aqueous solutions of target metals were prepared by dissolving chloride salt (analytical grade purchased from Sinopharm Chemical Reagent) of the respective metals in hydrochloric acid. 1-(3-Aminopropyl) imidazole ( $\geq 97 \%)$, purchased from Aldrich Chemicals; methyl isothiocyanate (97\%); 1-butylimidazole (97\%), purchased from Suiyuan Chemical Technology (Shanghai) Ltd; 1-bromobutane (99\%), 1-bromohexane (99\%), 1-bromooctane (99\%) from Shanghai Civic Chemical Technology Co., Ltd; Acetonitrile (HPLC grade) from Labing Chemical; Carbon tetrachloride, purchased from Aladdin Chemical Company.

\subsection{Physical measurements}

To analyze the extraction efficiency of metal ions from the aqueous sample solution, metal ions concentration was determined by inductively coupled plasma atomic emission spectrometry (ICP-AES, Varian 700-ES series) measurements of cadmium, copper, and zinc with the wavelengths: $324.75 \mathrm{~nm}$ (Cu), $213.86 \mathrm{~nm}(\mathrm{Zn}), 228.80 \mathrm{~nm}$ (Cd), respectively. The UV-visible spectra of metal solutions were recorded on visible spectrophotometer PTS 2000 in the range of 200-1000 $\mathrm{nm}$. The water content of ionic liquids was measured by a Mettler-Toledo DL39 Karl Fischer coulometer. The solubility of ionic liquids in the aqueous phase was determined by the Shimadzu TOC-V $\mathrm{CHP}$ analyzer operating between 40 to $800{ }^{\circ} \mathrm{C}$. Element analyses were carried out on a Perkin-Elmer $2400 \mathrm{C}, \mathrm{H}$, and $\mathrm{N}$ element analyzer.

The ionic liquids were dried in vacuum under anhydrous phosphorus pentoxide and stored in desiccators to guarantee the long-term stability of ionic liquids.

\subsection{Synthesis}

The Ionic liquids 1-Hexyl-3-methylimidazolium hexafluorophosphate, [HMPSM][PF6] (IL1) and 1-Butyl-3- 
methylimidazolium hexafluorophosphate, [BMPSM][PF 6 ] (IL2) were synthesized followed by metathesis route.

For synthesis, equal molar amounts of 1-(3-Aminopropyl) imidazole and butyl bromide in three mouthed flasks fixed with a reflux condenser were refluxed for $24-72 \mathrm{~h}$ at $70^{\circ} \mathrm{C}$ with intelligent magnetic stirring until two phases formed. The upper phase was gradually poured and added acetonitrile followed by scrupulous mixing. The acetonitrile was decanted and the procedure repeated twice to make sure that unreacted materials were removed from the lower phase. The resulting liquid, [BMPSM] [Br] was poured from the reaction vessel to a plastic bottle followed by the addition of $500 \mathrm{~mL}$ of deionized water.

$\mathrm{KPF}_{6}$ was added slowly in the resulting liquid solution to prevent the reaction temperature of solution to rise. After adding $\mathrm{KPF}_{6}$ solution stratification observed, removing the upper layer solution phase and then constantly washing the aqueous phase with distilled water until added to $\mathrm{AgNO}_{3}$ without precipitation, which showed that the $\mathrm{KBr}$ has been produced in the reaction. The final product, i.e. [BMPSM] $\left[\mathrm{PF}_{6}\right]$ ionic liquid was carried out by removing the water with the help of rotary evaporation. The purity or yield of ionic liquid was $83.79 \%$ and it confirmed by measuring the water content using Karl Fischer coulometer. The chemical reaction route shown in Figure 1.

$$
[\mathrm{BMPSM}]^{+}[\mathrm{Br}]^{-}+\mathrm{KPF}_{6} \stackrel{40^{\circ} \mathrm{C}, 24 h}{\longrightarrow}[\mathrm{BMPSM}]^{+}\left[\mathrm{PF}_{6}\right]^{-}+\mathrm{KBr}
$$

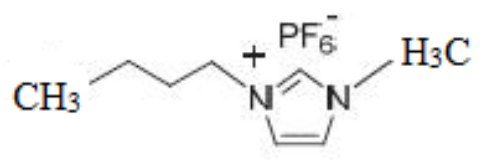

\section{$[\mathrm{BMPSM}]^{+}\left[\mathrm{PF}_{6}{ }^{\top}\right.$}

Figure 1. 1-Butyl-3-methylimidazolium hexafluorophosphate ionic liquid

The hexyl derivative was prepared in similar manner and also all the parameters remained the same as described (Figure 2) and the purity or yield of ionic liquid was $74.53 \%$.

$\left[\mathrm{HMPSM}^{+}[\mathrm{Br}]^{-}+\mathrm{KPF}_{6} \stackrel{40^{\circ} \mathrm{C}, 24 \mathrm{~h}}{\longrightarrow}\left[\mathrm{HMPSM}^{+}\left[\mathrm{PF}_{6}\right]^{-}+\mathrm{KBr}\right.\right.$

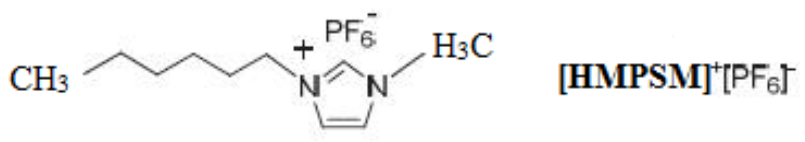

Figure 2. 1-Hexyl-3-methylimidazolium hexafluorophosphate ionic liquid

\subsection{Liquid-liquid extraction of metal ions}

The sample solutions used for the extraction experiment were prepared by dissolving solid $\mathrm{ZnCl}_{2}, \mathrm{CuCl}_{2}$, and $\mathrm{CdCl}_{2}$ in deionized water. A certain volume $(0 \mathrm{~mL}-5 \mathrm{~mL})$ of mixtures of solutions was taken and well shaken for one hour with $0.1 \mathrm{~mL}$ of a pure ionic liquid. Each mixture was shaken vigorously to facilitate the transfer of compounds into the ionic liquid phase. Afterwards, the mixture was centrifuged to separate aqueous phase and ionic liquid phase. The phases were then separated for analysis; the composition of aqueous phase was analyzed by inductively coupled plasma atomic emission spectrometry (ICP-AES). The metal ion concentration in the ionic liquid phase was deduced from difference between initial concentration of metal ions in aqueous phase and concentration of metal ions in the raffinate.

The efficiency of the extraction process was evaluated by the calculation of the extraction percentage rate $(\% E)$ using the following equation:

$$
\% E=100 * \frac{\left(C_{\text {in }}-C_{\text {fin }}\right)}{C_{\text {in }}}
$$

Where $C_{\text {in }}(\mathrm{mg} / \mathrm{L})$ is the initial concentration of the aqueous solution and $C_{\text {fin }}(\mathrm{mg} / \mathrm{L})$ is the content of metal ions in the raffinate phase after extraction. The extraction percentage was determined at $303 \mathrm{~K}$. The experiments are conducted trice to make sure the repeatability of experiments, and mean values are reported. The reproducibility of the assay, as measured by relative standard deviation, was $3 \%$ or less.

\section{Results and discussion}

The extraction percentage of metal ions from the hydrochloride aqueous solution in the ionic liquid phase at $303 \mathrm{~K}$ was determined. For this purpose, [BMPSM] $\left[\mathrm{PF}_{6}\right]$ and [HMPSM][PF6] ionic liquids were chosen on the basis of their low miscibility in water.

\subsection{Extraction of metal ions}

The extraction effects of ionic liquids for selected metal ions were determined by taking $1 \mathrm{~mL}$ of [HMPSM] [PF 6 ] (IL1) \{also same for [BMPSM][PF6] (IL2)\} mixed with $50 \mathrm{~mL}$ of 20 $\mathrm{mg} / \mathrm{L}$ zinc chloride, copper chloride and cadmium chloride solution at room temperature for a certain period of time. The solution was separated into two-phase (biphasic) after the extraction effects and then concentrations of remaining metal ions in the water were measured.

The experimental results showed that the ionic liquid has certain extraction effects on $\mathrm{Cd}$ and $\mathrm{Cu}$ also. The extraction rate is shown in Figure 3 below:

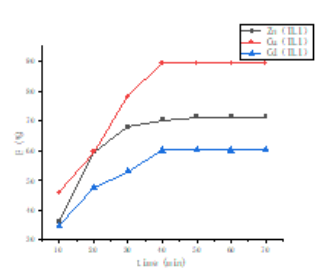

Figure 3a

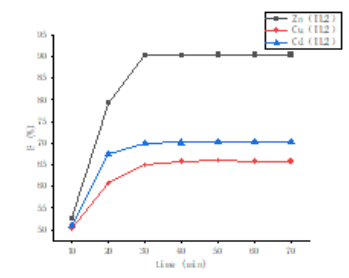

Figure $3 b$
Figure 3. Comparison of extraction effects of IL1 (Figure 3a) and IL2 (Figure 3b)

As shown in Figure 3a, IL1 has better extraction effect on copper metal ions and its extraction efficiency reached $83 \%$, while the extraction rate on cadmium and zinc ions is relatively less i.e. $65 \%$ and $72 \%$ respectively.

IL2 showed better extraction effects on $\mathrm{Zn}$ metal ions and its extraction efficiency reached $91 \%$ as shown in Figure $3 b$. 
The extraction efficiency of $\mathrm{Cu}$ reached $72 \%$, and the extraction rate of $\mathrm{Cd}$ reached $65 \%$ by using IL2. The extraction effect of these two metal ions is less than that of $\mathrm{Zn}$, which proves that IL2 can be used for selective extraction of $\mathrm{Zn}$ from water. The extraction rate on $\mathrm{Cu}$ metal ions with IL2 is not as good as IL1.

The extraction effects on metal ions were compared under the same experimental conditions for IL1 and IL2, and the results are shown in Figure 4.

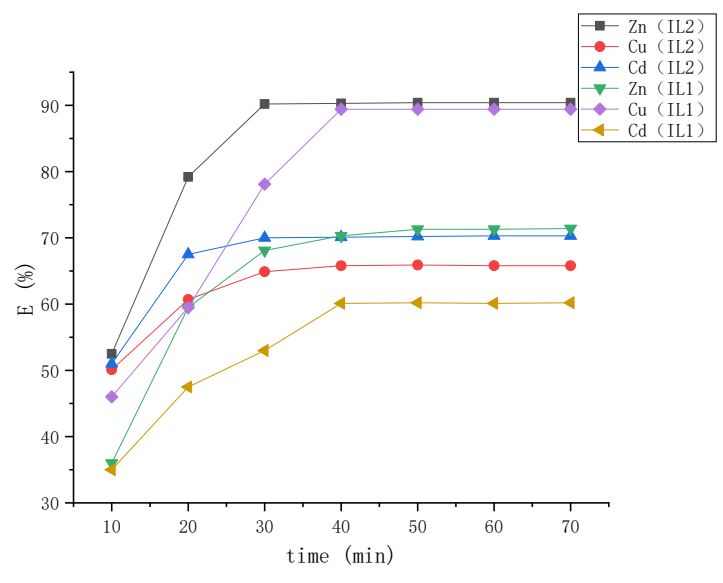

Figure 4. Extraction effect of ionic liquid (IL1) and (IL2) on metal ions

The contact time of IL1 with zinc metal ions aqueous solution started from 0 to 70 minutes, but after 10 minutes IL1 began to show extraction effects towards zinc metal ions and continued till 50 minutes and that time extraction rate was $70 \%$. After 50 minutes extraction effects became constant until the experiment finished. However, the contact time of IL2 for zinc ions was also 0 to 70 minutes but after 20 minutes, extraction effects were outstanding from IL2 that reached $80 \%$ and continued to illustrated considerable extraction effects until reached at $90 \%$ within 30 minutes of the experiment. After 30 minutes, the extraction efficiency was constant and stable up to $90 \%$. The extraction rate increased continuously with increasing time. After that, the extraction rate no longer significantly increased with the increase of time span and finally stabled at about $90 \%$, which means IL2 has higher extraction rate and efficiency than IL1. Based on extraction efficiency results, IL2 may be considered for further extraction experiments for selected metal ions.

\subsection{Analysis of ionic liquid (IL2)}

During the synthesis process of ionic liquid, the purity of the ionic liquid (83\%) is not high due to the large microwave power (700W), and some of the conventional ionic liquids change the cohesive force, the process as shown in Figure 5.

Enhanced mass transfer can effectively impede the adverse effects of steric hindrance, in that way promoting the extraction of zinc metal ions. Ultrasonic waves, stirring strength and other factors can reduce the hydration radius of metal ions in water and improve the mass transfer rate.
The issue of cohesion and steric hindrance needs further study.

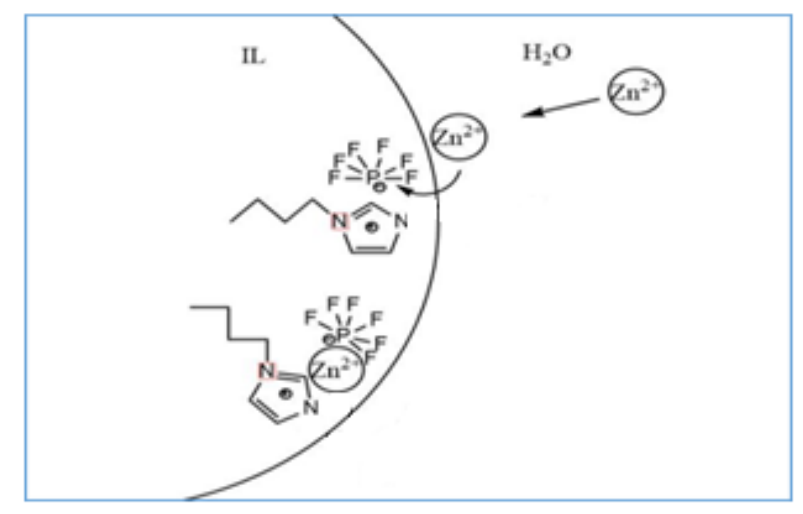

Figure 5. The extraction process of ILS

Influential factor on $\mathrm{Zn}$ metal ion extraction rateThe adaptability of extraction effect is an important indicator to measure the quality of an extractant. The extraction effect of ILs may be affected by the extraction conditions. Therefore, the stability of IL2 extraction on Zn metal ions under different extraction conditions was studied and the adaptability results of extraction circumstances were investigated.

Effect of ultrasonic wave Ultrasonic waves are often used to enhance mass transfer because of their strong energy as an auxiliary means of extraction. The extraction of $\mathrm{Zn}$ from water, ultrasound will considerably affect the extraction effect of ionic liquids. The adaptability of the extraction effect under different intensity ultrasonic waves on zinc ion extraction is as Figure 6 follows:

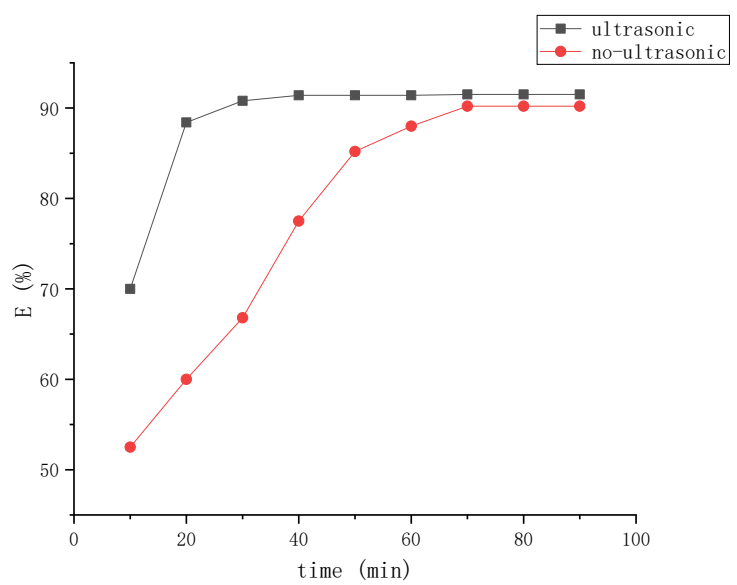

Figure 6. Effect of ultrasonic waves on the extraction rate of IL2 on $\mathrm{Zn}$

As shown in the figure, under the of ultrasonic waves enhanced the mass transfer rate, the extraction rate was greatly accelerated and the extraction time reduced from $70 \mathrm{~min}$ to $30 \mathrm{~min}$, and more importantly, the final extraction rate also increased from $88 \%$ to $94.4 \%$. It is speculated that the hydration of metal ions by ultrasonic waves has a certain reduction effect so that $\mathrm{Zn}$ metal ions in the mass transfer process is less susceptible to steric 
hindrance effects so that the extraction of IL2 have high adaptability on different ultrasound waves.

\section{Conclusion}

In this work, preliminary consequences on the extraction of $\mathrm{Zn}, \mathrm{Cd}$, and $\mathrm{Cu}$ from the aqueous hydrochloride solutions in ionic liquids in the absence of chelating agents are shown. The composition of ionic liquids used as extraction agents were seen to strongly influence efficiency of the extraction process. With [BMPSM] $\left[\mathrm{PF}^{6}\right]$ ionic liquid, almost complete extraction of $\mathrm{Zn}$ metal ions has been achieved. Furthermore, it was also found that the extraction rate improved with the impact of the ultrasound wave factor.

This work demonstrates the promising potential of ionic liquids to be used as extraction agents in green extraction processes since it is possible to design a specific ionic liquid for each metal ion by changing either anion or the cation to create an efficient process.

\section{Conflict of Interest}

The authors declare that there is no conflict of interest regarding the publication of this article.

\section{Acknowledgment}

This research was completed with the support of the National Natural Science Foundation of China (Grant No.51574238) and the University Student Innovation Training Program of China (Grant No.20181030).

\section{References}

Babel S. and Kurniawan T.A. (2004), Cr(VI) removal from synthetic wastewater using coconut shell charcoal and commercial activated carbon modified with oxidizing agents and/or chitosan, Chemosphere, 54(7), 951-967. https://doi.org/10.1016/j.chemosphere.2003.10.001.

Blais J.F., Dufresne S. and Mercier G. (1999), State of the art of technologies for metal removal from industrial effluents, Journal of Water Science, 12(4), 687-711.

Caparica R., Júlio A., Baby A.R., Araújo M.E.M., Fernandes A.S., Costa J.G., Santos de Almeida T. (2018), Choline-amino acid ionic liquids as green functional excipients to enhance drug solubility, Pharmaceutics, 10(4), 288.

Faur-Brasquet C., Kadirvelu K. and Le Cloirec, P. (2002), Removal of metal ions from aqueous solutions by adsorption: competition with organic matter, Carbon, 40, 2387-2392.

Fu F. and Wang Q. (2011), Removal of heavy metal ions from wastewaters: A review, Journal of Environmental Management, 92(3), 407-418. https://doi.org/10.1016/ j.jenvman.2010.11.011.

Gardas R.L. and Coutinho A.P. (2008), A group contribution method for viscosity estimation of ionic liquids, 266, 195-201. https://doi.org/10.1016/j.fluid.2008.01.021.

Germani R., Mancini V., Savelli G. and Spreti N. (2007), Mercury extraction by ionic liquids: temperature and alkyl chain length effect, Tetrahedron Letters, 48, 1767-1769. https://doi.org/10.1016/j.tetlet.2007.01.038.

Ghandi K. (2018), A Review of Ionic Liquids, Their Limits and Applications. May. https://doi.org/10.4236/gsc.2014.41008.

Gunatilake S.K. (2015), Methods of removing heavy metals from industrial wastewater, Multidisciplinary Engineering Scinece Studies (JMESS), 1(1), 12-18. www.jmess.org.
He J., Yang J., Sarwar M.T., Duan C. and Zhao Y. (2020), Comparative investigation on copper leaching efficiency from waste mobile phones using various types of ionic liquids, Journal of Cleaner Production, 256, 120368. https://doi.org/10.1016/j.jclepro.2020.120368.

Isosaari P., Srivastava V. and Sillanpää M. (2019), Ionic liquidbased water treatment technologies for organic pollutants: Current status and future prospects of ionic liquid mediated technologies, Science of the Total Environment, 690, 604-619. https://doi.org/10.1016/j.scitotenv.2019.06.421.

Kenntner N., Krone O., Altenkamp R. and Tataruch F. (2003), Environmental contaminants in liver and kidney of freeranging Northern Goshawks (Accipiter gentilis) from three regions of Germany, Archives of Environmental Contamination and Toxicology, 45(1), 128-135. https://doi.org/10.1007/s00244-002-2100-8.

Larsson K. and Binnemans K. (2014), Selective extraction of metals using ionic liquids for nickel metal hydride battery recycling, Green Chemisty, 16(10), 4595-4603. https://doi.org/10.1039/C3GC41930D.

Li C. and Trost B.M. (2008), Green chemistry for chemical synthesis, 105(36), 13197-13202.

Marsousi S., Karimi-sabet J., Moosavian M.A. and Amini Y. (2019), Liquid-liquid extraction of calcium using ionic liquids in spiral micro fluidics, Chemical Engineering Journal, 356(August 2018), 492-505. https://doi.org/10.1016/ j.cej.2018.09.030.

Nordberg M. and Nordberg G.F. (2009), Toxicology and biological monitoring of metals. In General and Applied Toxicology, John Wiley \& Sons, Ltd. https://doi.org/10.1002/ 9780470744307.gat145.

Pacheco-Fernández I. and Pino V. (2019), Green solvents in analytical chemistry, Current Opinion in Green and Sustainable Chemistry, 18, 42-50.

Papaiconomou N., Svecova L., Bonnaud C., Cathelin L., Billard I. and Chainet E. (2015), Possibilities and limitations in separating $\mathrm{Pt}(\mathrm{IV})$ from $\mathrm{Pd}(\mathrm{II})$ combining imidazolium and phosphonium ionic liquids, Dalton Transactions, 44(46), 20131-20138. https://doi.org/10.1039/C5DT03791C.

Phuong T., Pham T., Cho C. and Yun Y. (2010), Environmental fate and toxicity of ionic liquids: A review. Water Research, 44(2), 352-372. https://doi.org/10.1016/j.watres.2009.09.030.

Quijada-Maldonado E., Sánchez F., Pérez B., Tapia R. and Romero J. (2018), Task-specific ionic liquids as extractants for the solvent extraction of molybdenum(VI) from aqueous solution using different commercial ionic liquids as diluents, Industrial \& Engineering Chemistry Research, 57(5), 1621-1629. https://doi.org/10.1021/acs.iecr.7b04147.

Ríos A.P.D.L., Hernández-Fernández F.J., Presa H., Gómez D. and Vílora G. (2009), Tailoring supported ionic liquid membranes for the selective separation of transesterification reaction compounds, 328, 81-85. https://doi.org/10.1016/j.memsci. 2008.11.041.

Rzelewska M., Baczyńska M., Wiśniewski M. and Regel-Rosocka M. (2017), Phosphonium ionic liquids as extractants for recovery of ruthenium(III) from acidic aqueous solutions, Chemical Papers, 71(6), 1065-1072. https://doi.org/ 10.1007/s11696-016-0027-1.

Sheldon R.A. (2001), Catalytic reactions in ionic liquids, Chemical Communications, 23, 2399-2407. https://doi.org/10.1039/ B107270F. 
Singh S.K. and Savoy A.W. (2020), Ionic liquids synthesis and applications: An overview, Journal of Molecular Liquids, 297, 112038. https://doi.org/10.1016/j.molliq.2019.112038.

Tian M., Fang L., Yan X., Xiao W. and Row K.H. (2019), Determination of heavy metal ions and organic pollutants in water samples using ionic liquids and ionic liquid-modified sorbents, Journal of Analytical Methods in Chemistry, 2019, 119. https://doi.org/10.1155/2019/1948965.

Toral A.R., de los Ríos A.P., Hernández F.J., Janssen M.H.A., Schoevaart R., van Rantwijk F. and Sheldon R.A. (2007), Crosslinked Candida antarctica lipase $B$ is active in denaturing ionic liquids, Enzyme and Microbial Technology, 40(5), 1095-1099. https://doi.org/10.1016/j.enzmictec.2006.08.027.

Vekariya R.L. (2017), A review of ionic liquids: Applications towards catalytic organic transformations, Journal of Molecular Liquids, 227, 44-60. https://doi.org/10.1016/ j.molliq.2016.11.123.

Wieszczycka K. (2013), Recovery of Zn(II) from multielemental acidic chloride solution with hydrophobic 3-pyridineketoxime, Separation and Purification Technology, 114, 17-23. https://doi.org/10.1016/j.seppur.2013.04.002.

Wieszczycka K., Kaczerewska M., Krupa M., Parus A. and Olszanowski A. (2012), Solvent extraction of copper(II) from ammonium chloride and hydrochloric acid solutions with hydrophobic pyridineketoximes, Separation and Purification Technology, 95, 157-164. https://doi.org/10.1016/j.seppur. 2012.04.025.

Wojciechowska A., Wieszczycka K. and Wojciechowska I. (2017a), $\mathrm{Pb}$ (II) removal with hydrophobic quaternary pyridinium salt and methyl isobutyl ketone, Hydrometallurgy, 171, 206-212. https://doi.org/10.1016/j.hydromet.2017.05.017.

Wojciechowska A., Wieszczycka K. and Wojciechowska I. (2017b), Efficient recovery of copper from aqueous solutions with pyridine extractants (oxime, ketone) and their quaternary pyridinium salts, Separation and Purification Technology, 185, 103-111. https://doi.org/10.1016/j.seppur.2017.05.020.

Zhang H., Zhu M., Zhao W., Li S. and Feng G. (2018), ScienceDirect molecular dynamics study of room temperature ionic liquids with water at mica surface, Green Energy and Environment, 3(2), 120-128. https://doi.org/10.1016/j.gee.2017.11.002. 